\title{
Troxerutin induces protective effects against ultraviolet B radiation through the alteration of microRNA expression in human HaCaT keratinocyte cells
}

\author{
KWANG SIK LEE ${ }^{1,2^{*}}$, HWA JUN CHA ${ }^{3 *}$, GHANG TAI LEE ${ }^{1,2}$, KUN KOOK LEE ${ }^{1}$, JIN TAE HONG ${ }^{2}$, \\ KYU JOONG AHN ${ }^{4}$, IN-SOOK AN ${ }^{3}$, SUNGKWAN AN ${ }^{3}$ and SEUNGHEE BAE ${ }^{3}$ \\ ${ }^{1}$ Songpa R\&D Center, Coreana Cosmetics Co., Ltd., Cheonan, Chungcheongnam-do 330-833; \\ ${ }^{2}$ College of Pharmacy and Medical Research Center, Chungbuk National University, Cheongju, Chungcheongbuk-do 361-763; \\ ${ }^{3}$ Molecular-Targeted Drug Research Center and Korea Institute for Skin and Clinical Sciences, Konkuk University; \\ ${ }^{4}$ Department of Dermatology, Konkuk University School of Medicine, Seoul 143-701, Republic of Korea
}

Received September 17, 2013; Accepted January 20, 2014

DOI: 10.3892/ijmm.2014.1641

\begin{abstract}
Ultraviolet light B (UVB), contained in sunlight, induces damaging effects on skin by impairing cells in the epidermis and dermis. In particular, keratinocytes in the epidermis are those cells which are mainly affected by UVB light. UVB radiation induces cell death, growth arrest, DNA damage and restricts cell migration. Various phytochemicals have been shown to alleviate UVB-induced cellular damage. Troxerutin is a natural flavonoid rutin mainly found in extracts of Sophora japonica, and is a well-known antioxidant and anti-inflammatory compound used in experimental mouse models. In this study, we examined the effects of troxerutin on UVB-induced damage in $\mathrm{HaCaT}$ cells. $\mathrm{HaCaT}$ cells were pretreated with troxerutin $(0-10 \mu \mathrm{M})$ and then exposed to UVB radiation $\left(50 \mathrm{~mJ} / \mathrm{cm}^{2}\right)$. Cell viability, cell cycle and migration assays were performed to determine the protective effects of troxerutin on the cells. DNA repair activity was also measured. Troxerutin protected the cells against UVB-induced damage, such as cell death, growth arrest, restriction of cell migration and decreased DNA repair activity in $\mathrm{HaCaT}$ cells. Analyses of microRNA (miRNA) expression demonstrated that the protective effects of troxerutin correlated with alterations in miRNA expression, as indicated by Gene Ontology analyses of putative target genes. Overall, our data demonstrate that
\end{abstract}

Correspondence to: Dr Seunghee Bae, Molecular-Targeted Drug Research Center and Korea Institute for Skin and Clinical Sciences, Konkuk University, 120 Neungdong-ro, Gwangjin-gu, Seoul 143-701, Republic of Korea

E-mail: sbae@konkuk.ac.kr

${ }^{*}$ Contributed equally

Key words: troxerutin, keratinocyte, ultraviolet B, microRNA, cell death troxerutin exerts protective effects against UVB-induced damage by regulating miRNA expression.

\section{Introduction}

Ultraviolet (UV) radiation found in sunlight is the major environmental cause of skin aging and skin disorders (1). There are three different wavelengths of UV radiation, subdivided as UVC (290-320 nm), UVB (290-320 nm) and UVA (320-400 nm) (2). UVC radiation contains the highest energy, which can induce skin damage. However, the majority of UVC radiation is blocked by the atmosphere. By contrast, UVA radiation is, on average, 1,000-fold lower in energy than UVB radiation and its transmission through the atmosphere is greater (3). UVB radiation reaches the dermis and epidermis when penetrating the atmosphere. UVB radiation is absorbed by the epidermis and dermis and causes damage to various cells and to the extracellular matrix of the skin (4-6). Keratinocytes are particularly influenced by UVB radiation due to their outermost cutaneous location (7). Keratinocytes, mainly contained in the epidermis, have been implicated in its development via differentiation (8). In keratinocytes, UVB radiation induces the formation of 'sunburn cells' (keratinocytes undergoing apoptosis) and may result in skin cancer (5). UVB induces keratinocyte apoptosis by promoting DNA damage, death receptor activation and the production of reactive oxygen species (ROS) (9). UVB radiation can stimulate UVB-induced signaling pathways involving p53 and MAP kinases (JNK and p38), and can alter gene expression and subsequently induce cell cycle arrest, apoptosis or cellular senescence (10-14). Recently, microRNAs (miRNAs) have been implicated in UVB responses in keratinocytes (15-16). The miRNA, miR-23a, regulates DNA damage repair and apoptosis by targeting topoisomerase-1, caspase-7 and serine/ threonine kinase 4 (STK4) (17). In addition, UVB radiation induces apoptosis by upregulating miR-141, which then leads to the suppression of phosphatase and tensin homolog (PTEN) in keratinocytes (18). Aberrantly expressed miRNAs have been linked to apoptosis and cell cycle arrest in UVB-exposed 
keratinocytes, where they can be used as regulators of UVB responses (19). In the current study, we identified a novel phytochemical that protect against UVB-induced cell death through the regulation of miRNA expression.

Troxerutin, \{vitamin P4; 3',4',7'-Tris[O-(2-hydroxyethyl)] rutin\} is a natural flavonoid rutin mainly found in extracts of Sophora japonica, and is a well-known antioxidant and anti-inflammatory compound used in experimental mouse models (20-24). In addition, troxerutin has been shown to improve capillary function by suppressing capillary fragility and abnormal leakage (25). It has been shown to inhibit erythrolysis and exert anti-thrombotic, fibrinolytic, edemaprotective and rheological effects in a model of chronic venous insufficiency (26).

In the present study, we demonstrate that troxerutin exerts protective effects against UVB radiation by regulating miRNA expression. The current data may enhance our understanding of the protective mechanisms of troxerutin in UVB-exposed skin.

\section{Materials and methods}

Cell culture and reagents. HaCaT cells, an immortalized human keratinocyte cell line, were grown as monolayers at $37^{\circ} \mathrm{C}$, in a $5 \% \mathrm{CO}_{2}$ atmosphere, in DMEM medium (GibcoInvitrogen, Carlsbad, CA, USA) supplemented with $10 \%$ fetal bovine serum (FBS; Sigma-Aldrich, St. Louis, MO, USA) and $1 \%$ penicillin/streptomycin solution (Gibco-Invitrogen). Viability assays were carried out in 96-well microplates seeded with $4 \times 10^{4} \mathrm{HaCaT}$ cells. Cell cycle analyses, DNA damage assays, and microarray analyses were performed in $60-\mathrm{mm}$ culture dishes seeded with $7 \times 10^{5} \mathrm{HaCaT}$ cells. Troxerutin was purchased from Sigma-Aldrich. Hydrogen peroxide (30\%) was purchased from BioShop Canada Inc. (Burlington, ON, Canada).

Exposure of HaCaT cells to UVB radiation. The HaCaT cells were exposed to UVB radiation using a G8T5E lamp (Sankyo Denki, Toshima-ku, Japan). The UVB radiation intensity was measured by a UV light meter (UV-340; Lutron, Taiwan, Taipei). The cells were washed and resuspended in phosphatebuffered saline (PBS) prior to exposure to UVB radiation. Non-exposed control samples were maintained in the dark under the same conditions. Following exposure to UVB radiation, the cells were grown in fresh medium.

Cell viability assay. Cell viability was measured by a water-soluble tetrazolium salt (WST-1) assay. HaCaT cells were seeded at $4 \times 10^{4}$ cells/well in 96-well microplates and incubated for $24 \mathrm{~h}$. In order to measure troxerutin toxicity, the cells were treated with the indicated concentrations of troxerutin for $24 \mathrm{~h}$. In order to determine the protective effects of troxerutin against UVB radiation, the cells were incubated with the indicated concentrations of troxerutin for 4,8 and $12 \mathrm{~h}$. The troxerutinpre-treated $\mathrm{HaCaT}$ cells were then exposed to $50 \mathrm{~mJ} / \mathrm{cm}^{2}$ of UVB radiation followed by incubation for $24 \mathrm{~h}$. WST-1 solution (EZ-Cytox Cell Viability Assay kit; ITSBio, Seoul, Korea) was then added to the cells for $1 \mathrm{~h}$. The absorbance of each sample was measured using a microplate reader (iMark; Bio-Rad Laboratories, Hercules, CA, USA) with filters at $450 \mathrm{~nm}$ and a reference wavelength at $620 \mathrm{~nm}$. The results were presented as the relative absorbance [optical density (OD)] compared with untreated HaCaT cells.

Cell cycle analysis. Cell cycle distribution was measured by propidium iodide (PI; Sigma-Aldrich) staining. HaCaT cells were seeded at a density of $7 \times 10^{5}$ cells in $60-\mathrm{mm}$ culture dishes, and incubated for $24 \mathrm{~h}$. The cells were pre-treated with $5 \mu \mathrm{M}$ troxerutin for $8 \mathrm{~h}$, and the troxerutin-pre-treated $\mathrm{HaCaT}$ cells were then exposed to $50 \mathrm{~mJ} / \mathrm{cm}^{2}$ of UVB radiation and incubated for $24 \mathrm{~h}$. The cells were fixed in $70 \%$ ethanol at $4^{\circ} \mathrm{C}$ for $3 \mathrm{~h}$, and stained with PI staining solution containing $50 \mu \mathrm{g} /$ ml PI (Sigma-Aldrich), 0.5\% Triton X-100 and $100 \mu \mathrm{g} / \mathrm{ml}$ RNase (both from BioShop Canada Inc.) at $37^{\circ} \mathrm{C}$ for $1 \mathrm{~h}$. The fluorescence intensity of each cell sample was detected by the FL2-H channel of the FACSCalibur flow cytometer (BD Biosciences, San Jose, CA, USA).

Migration assay. Migration activity in the cells was measured by a scratch wound assay. The HaCaT cells were seeded at a density of $7 \times 10^{5}$ cells in $60-\mathrm{mm}$ culture dishes, and cultured to $>90 \%$ confluency. The cells were pre-treated with $5 \mu \mathrm{M}$ troxerutin for $8 \mathrm{~h}$, and the troxerutin-pre-treated $\mathrm{HaCaT}$ cells were then exposed to $50 \mathrm{~mJ} / \mathrm{cm}^{2}$ of UVB radiation and scratched using a $200 \mu \mathrm{l}$ micropipette tip. At 0 and $48 \mathrm{~h}$, images were acquired of the scratch wound using a phase contrast microscope.

DNA repair activity assay. DNA repair activity was measured by using an impaired plasmid. HaCaT cells were seeded at a density of $7 \times 10^{5}$ cells in $60-\mathrm{mm}$ culture dishes, and incubated for $24 \mathrm{~h}$. The cells were transiently transfected with damaged (due to impaired exposure response to UVB) pGL3 Luciferase reporter vectors. $\mathrm{pSV}$ - $\beta$-galactosidase is a positive control vector that was used for monitoring the transfection efficiency. The transfected cells were pre-treated with $5 \mu \mathrm{M}$ troxerutin for $24 \mathrm{~h}$. After $24 \mathrm{~h}$, luciferase activity was measured using the Luciferase assay system (Promega, Fitchburg, WI, USA) as described in the instruction manual. The normalized results were presented as relative percentages of the experimental control values.

Detection of global miRNA expression levels. Alterations in global miRNA expression levels were measured using a SurePrint G3 Human v16.0 miRNA microarray kit (based on the miRBase release 19.0; Agilent Technologies, Santa Clara, CA, USA). Total RNA was extracted using TRIzol reagent (Invitrogen) according to the manufacturer's instructions, while RNA stability and purity were measured using the Bioanalyzer 2100 (Agilent Technologies), and calculated as $\mathrm{A}_{260 / \mathrm{A} 280}$ and $\mathrm{A}_{260 / \mathrm{A} 230}$ ratios using a MaestroNano Spectrophotometer (Maestrogen, Las Vegas, NV, USA). The total RNA eliminated phosphate was measured using calf intestine alkaline phosphatase (CIP), and was then labeled with pCp-Cy3, using T4 RNA ligase (all from Agilent Technologies). The labeled total RNA was hybridized to the miRNA microarray. Microarrays were scanned using an Agilent microarray scanner. The miRNA microarray data were normalized and analyzed using GeneSpring GX version 11.5 software (Agilent Technologies). 


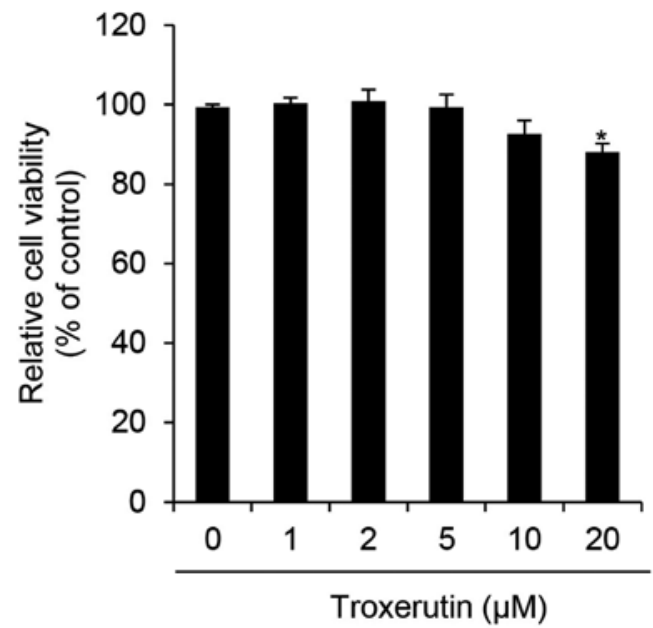

Figure 1.Effects of troxerutin on $\mathrm{HaCaT}$ cell viability. HaCaT cells $\left(4 \times 10^{4}\right.$ cells $)$ were seeded in 96-well plates and treated with indicated concentrations of troxerutin for $24 \mathrm{~h}$. Cell viability was measured by the water-soluble tetrazolium salt (WST-1) assay as described in Material and methods. The results are presented as the means \pm standard error (SE) of the percentage of the control optical density (OD) in triplicate. ${ }^{*} \mathrm{p}<0.05$.

miRNA target gene prediction and Gene Ontology (GO) analysis. A list of target genes for the selected miRNAs was extracted by TargetScan (http://www.targetscan.org). The biological functions of putative target genes were classified into different biological processes (GO categories). Enrichment analysis of GO categories was performed using DAVID Bioinformatics Resources 6.7 (http://david.abcc.ncifcrf.gov/). We selected significant $\mathrm{GO}$ categories using several parameters (count $>600$ and $p$-value $<0.01$ ).

Statistical analysis. Statistical significance was determined using a Student's t-test. Values of $p<0.05$ were considered to indicate statistically significant differences.

\section{Results}

Troxerutin reduces UVB-induced cytotoxicity in HaCaT cells. We first determined the cytotoxicity of troxerutin in $\mathrm{HaCaT}$ cells by performing WST-1 assays. The HaCaT cells were treated with the indicated concentrations of troxerutin and then incubated for $24 \mathrm{~h}$. Cytotoxicity in $\mathrm{HaCaT}$ cells was significantly decreased at $20 \mu \mathrm{M}$ troxerutin (Fig. 1). In a previous study, troxerutin was shown to exert limited, if any, cytotoxic effects at a dose of $20 \mu \mathrm{g} / \mathrm{ml}$ (approximately $26.93 \mu \mathrm{M}$ ) in V79 Chinese hamster lung fibroblasts (27). Therefore, a similar concentration range of troxerutin $(0-10 \mu \mathrm{M})$ was used in the current study. To determine whether troxerutin protects against UVB-induced growth arrest in $\mathrm{HaCaT}$ cells, we performed cell viability assays. The HaCaT cells were pre-treated with the indicated concentrations of troxerutin for 4,8 and $12 \mathrm{~h}$, and then exposed to $50 \mathrm{~mJ} / \mathrm{cm}^{2}$ of UVB radiation and incubated for $24 \mathrm{~h}$. Troxerutin prevented UVB-mediated growth arrest in a dose-dependent manner (Fig. 2). Troxerutin (5 $\mu \mathrm{M})$ effectively protected the cells against UVB-mediated growth arrest. Notably, pre-treatment with $5 \mu \mathrm{M}$ troxerutin for $8 \mathrm{~h}$ increased cell viability by $20.27 \%$ compared with untreated

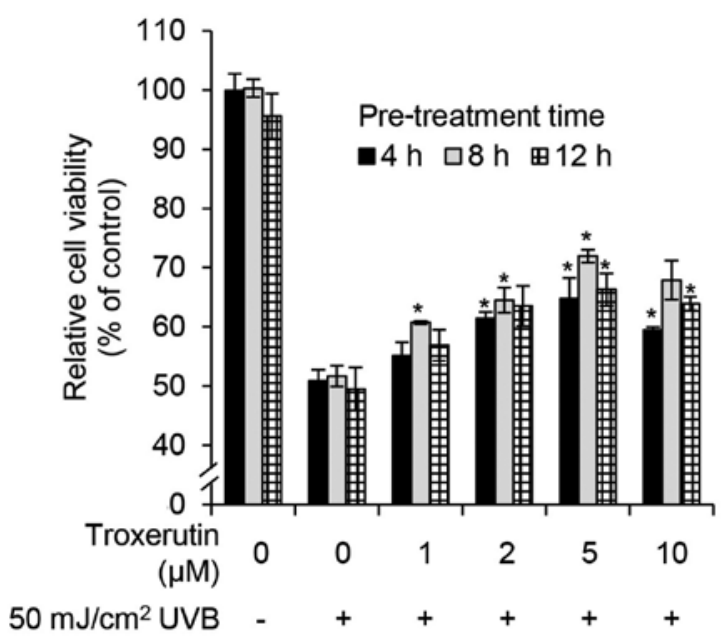

Figure 2. Protective effects of troxerutin on cell viability in ultraviolet $B$ (UVB)-exposed HaCaT cells. HaCaT cells $\left(4 \times 10^{4}\right.$ cells) were seeded in 96-well plates, and pre-incubated with indicated concentrations of troxerutin for 4,8 and $12 \mathrm{~h}$. Following pre-treatment, the HaCaT cells were exposed to $50 \mathrm{~mJ} / \mathrm{cm}^{2}$ of UVB and incubated for $24 \mathrm{~h}$. Cell viability was measured by the water-soluble tetrazolium salt (WST-1) assay as described in Materials and methods. Results are presented in triplicate, as the means $\pm \mathrm{SE}$ of the percentage of the control optical density (OD). Asterisks denote a significant difference compared with HaCaT cells treated with UVB alone $\left({ }^{*} \mathrm{p}<0.05\right)$

UVB-exposed HaCaT cells (Fig. 2). Overall, pre-treatment with troxerutin protected the cells from UVB-mediated growth arrest.

Troxerutin reduces UVB-induced death in HaCaT cells. As growth arrest and cell death often cause a decrease in cell viability, in the current study, we determined whether the troxerutin-mediated protective effects against UVB exposure were due to the suppression of growth arrest and cell death. In normal $\mathrm{HaCaT}$ cells, $50 \mathrm{~mJ} / \mathrm{cm}^{2}$ of UVB radiation caused an increase in the number of cells in the sub-G1 phase (1.49-11.49\%). However, in the $\mathrm{HaCaT}$ cells pre-treated with troxerutin, the UVB-mediated increase in the number of sub-G1 phase cells decreased to $4.51 \%$ (Fig. 3). As the sub-G1 phase indicates a population of dead cells, the troxerutin-mediated recovery of cell viability may involve the blockade of cell death-associated pathways.

Troxerutin enhances migration activity in HaCaT cells. We then determined whether troxerutin affects migration activity in $\mathrm{HaCaT}$ cells. To assess migration activity, the scratched wound healing assay was used as described in Materials and methods. The migration activity was increased by treatment with troxerutin in $\mathrm{HaCaT}$ cells as compared to the untreated cells. Exposure to UVB radiation attenuated migration in $\mathrm{HaCaT}$ cells, whereas troxerutin restored the migration activity to the control levels (Fig. 4).

Troxerutin induces DNA repair activity in HaCaT cells. To determine whether troxerutin increases DNA repair activity, we examined its effects using the damaged luciferase reporter vector. Troxerutin had no effects on luciferase activity in non-damaged pGL3 vector-transfected $\mathrm{HaCaT}$ cells (Fig. 5). 


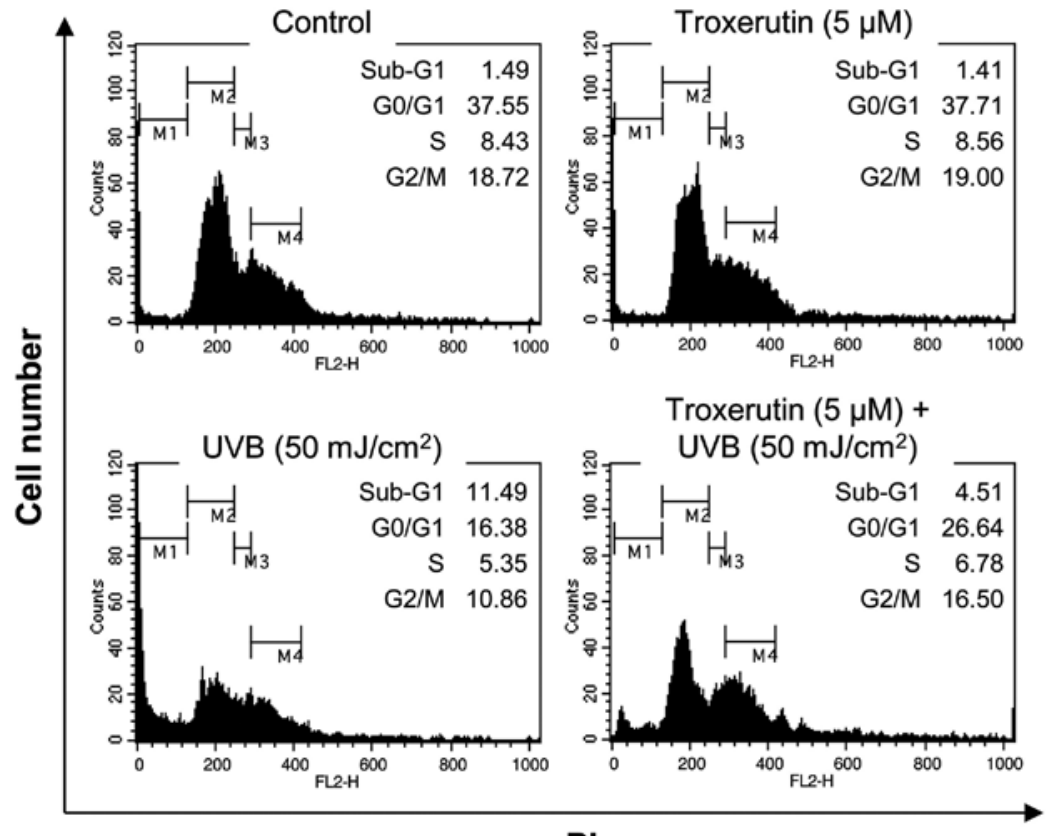

PI

Figure 3. Protective effects of troxerutin on cell cycle in ultraviolet B (UVB)-exposed HaCaT cells. HaCaT cells (7x10 $0^{5}$ cells) were seeded in 60 -mm culture dishes and pre-incubated with $5 \mu \mathrm{M}$ troxerutin for $8 \mathrm{~h}$. Following pre-treatment, the $\mathrm{HaCaT}$ cells were exposed to $50 \mathrm{~mJ} / \mathrm{cm}^{2}$ of UVB and incubated for $24 \mathrm{~h}$. The cell cycle was measured by propidium iodide (PI) staining. Cell distribution measured by fluorescence intensity in the sub-G1 phase is presented as a histogram plot. Each cell cycle population (G1, S, G2/M phase) was measured using gates.

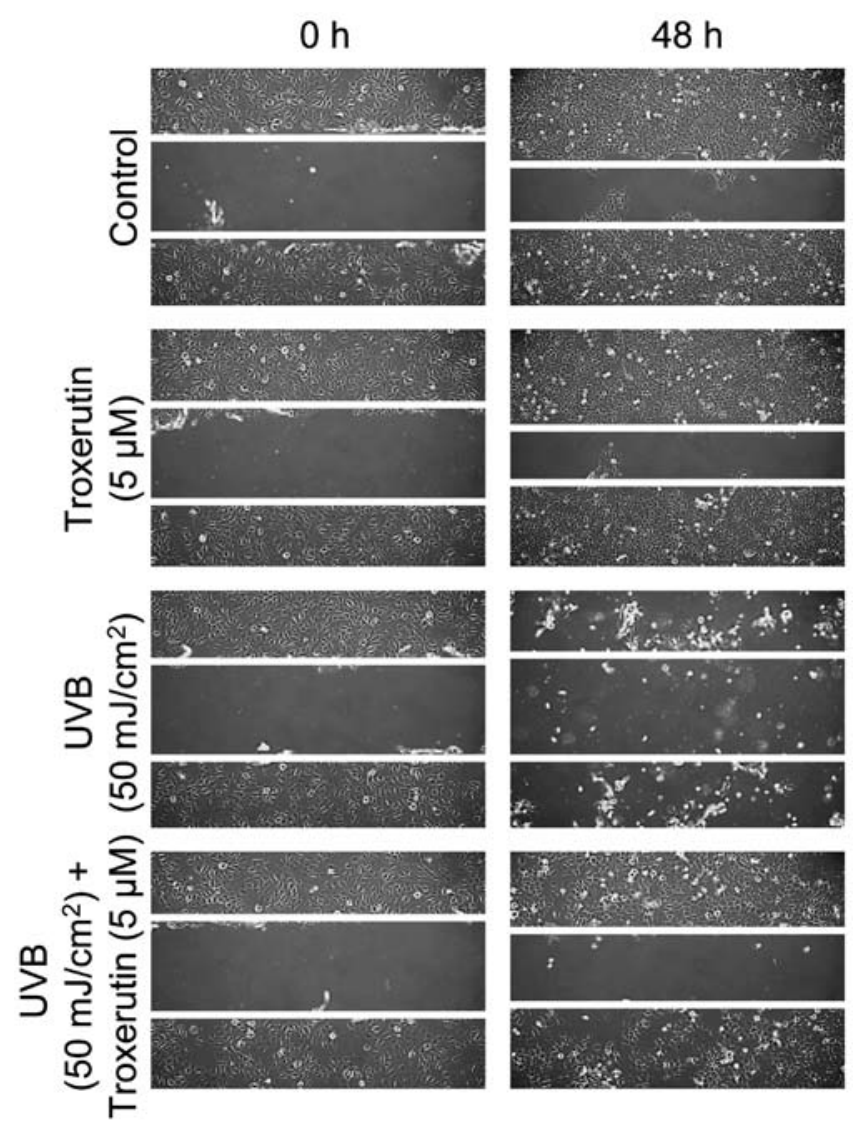

Figure 4. Protective effects of troxerutin on migration in ultraviolet B (UVB)exposed HaCaT cells. HaCaT cells $\left(7 \times 10^{5}\right.$ cells) were seeded in $60-\mathrm{mm}$ culture dishes, and pre-incubated with $5 \mu \mathrm{M}$ troxerutin for $8 \mathrm{~h}$. Following pretreatment, the HaCaT cells were exposed to $50 \mathrm{~mJ} / \mathrm{cm}^{2}$ of UVB and scratched using a pipet tip. Cells were then incubated for $48 \mathrm{~h}$. The left panel shows a representative image of indicated conditions immediately after scratching $(0 \mathrm{~h})$. The right panel shows a representative image of indicated conditions at $48 \mathrm{~h}$ after scratching.

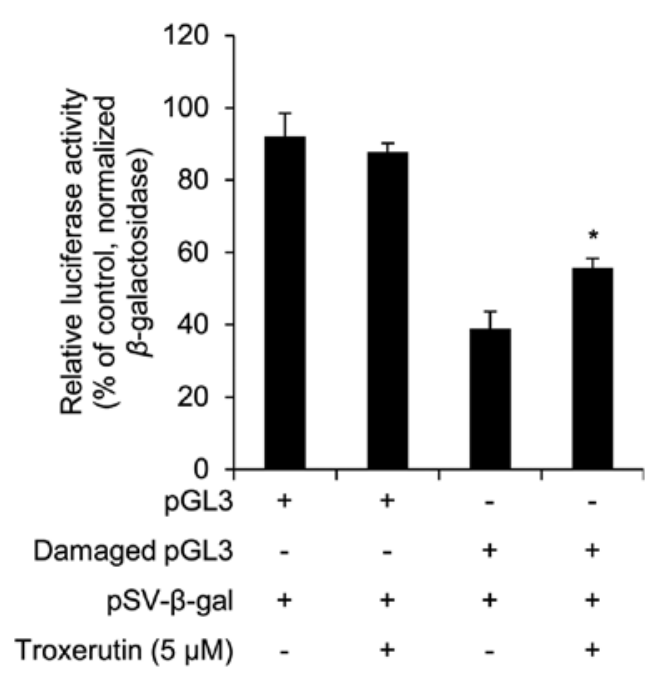

Figure 5. Protective effects of troxerutin on DNA repair activity. HaCaT cells $\left(7 \times 10^{5}\right.$ cells) were seeded in $60-\mathrm{mm}$ culture dishes and transfected with damaged pGL3 and pSV- $\beta$-galactosidase. Following transfection, the cells were pre-incubated with $5 \mu \mathrm{M}$ troxerutin for $8 \mathrm{~h}$. Following pre-treatment, the $\mathrm{HaCaT}$ cells were exposed to $50 \mathrm{~mJ} / \mathrm{cm}^{2}$ of ultraviolet B (UVB) and incubated for $24 \mathrm{~h}$. DNA repair activity is presented as luciferase activity. The results are presented in triplicate, as the means \pm standard error (SE) of the percentage of luciferase activity. Asterisks denote a significant difference compared with damaged $\mathrm{HaCaT}$ cells transfected with pGL3 alone ( $\left.{ }^{*} \mathrm{p}<0.05\right)$.

However, in the damaged-pGL3 vector-transfected $\mathrm{HaCaT}$ cells, troxerutin increased luciferase activity (Fig. 5). The increased luciferase activity was used to measure DNA repair activity; troxerutin increased DNA repair activity in $\mathrm{HaCaT}$ cells. Similarly, in a previous study, Maurya et al (28) demonstrated that troxerutin exerted protective effects against gamma radiation-induced damage in mouse cellular DNA. 


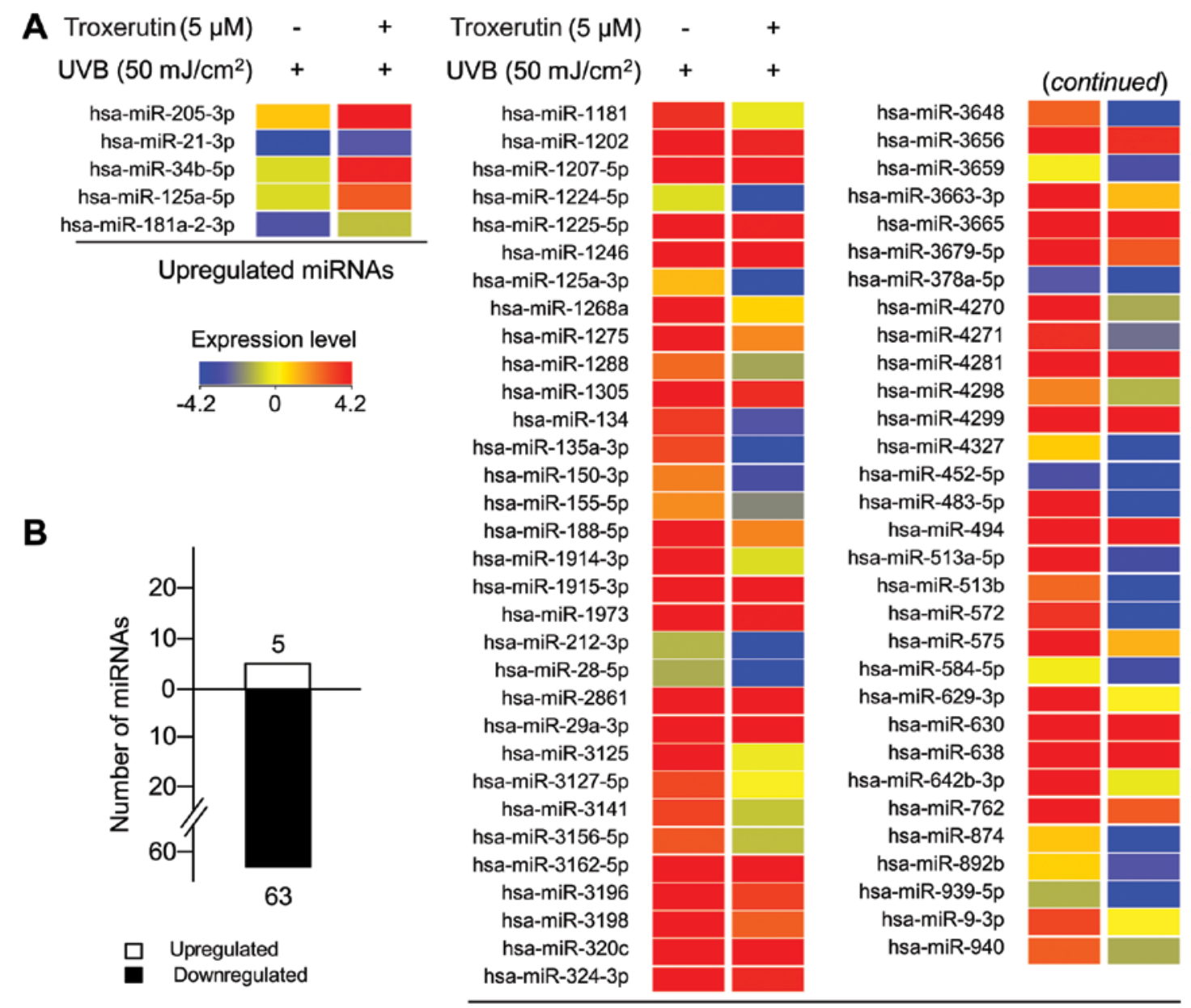

Downregulated miRNAs

Figure 6. Troxerutin-induced expression profiles of microRNAs (miRNAs) in ultraviolet B (UVB)-exposed HaCaT cells. (A) Heat map analysis of miRNAs upregulated and downregulated with a $\geq 2$-fold change in expression in UVB-irradiated HaCaT cells. HaCaT cells $\left(7 \times 10^{5}\right.$ cells) were seeded in 60 -mm culture dishes, and pre-incubated with $5 \mu \mathrm{M}$ troxerutin for $8 \mathrm{~h}$. Following pre-treatment, the $\mathrm{HaCaT}$ cells were exposed to $50 \mathrm{~mJ} / \mathrm{cm}^{2}$ of $\mathrm{UVB}$ and incubated for $24 \mathrm{~h}$. miRNA expression was determined using the SurePrint G3 Human v16.0 miRNA microarray kit. The color bars displaying fluorescence intensity correspond to each miRNA expression. Highly expressed miRNAs are shown in red, while those present at low levels are shown in blue. (B) Total number of miRNAs upregulated and downregulated by troxerutin.

Troxerutin alters miRNA expression profiles in UVB-exposed HaCaT cells. As described in the Introduction, miRNAs play a crucial role in UVB responses through specific binding to target genes (19). Therefore, in UVB-exposed $\mathrm{HaCaT}$ cells, we examined the effects of troxerutin on global miRNA expression levels. To analyze the global miRNA expression levels and identify putative alterations, we used miRNA gene microarrays, which contained 1,368 probes representing 1,205 human miRNAs. Total RNA, labeled with Cy3, a green fluorescent dye, was extracted from the $\mathrm{HaCaT}$ cells treated with or without troxerutin and then exposed to UVB radiation. Each Cy3-labeled sample was hybridized onto an miRNA microarray. Using analytical software (GeneSpring GX version 11.5; Agilent), we first normalized the data from each sample using global normalization. To obtain refined data, we did not take into consideration the absent and undetectable data in all samples. Lastly, significantly altered miRNAs were designated as miRNAs with a $\geq 2$-fold change in expression. A total of 68 miRNAs, 5 upregulated miRNAs and 63 downregulated miRNAs, were selected for analyses (Fig. 6 and Table I). miR-205-3p expression was increased by 4.29 -fold, whereas
miR-513b, miR483-5p and miR-3648 expression was significantly decreased by $23.10-, 16.61$ - and 11.55 -fold, respectively. Overall, our results demonstrate that troxerutin protects $\mathrm{HaCaT}$ cells from UVB-induced damage through the specific regulation of miRNA expression.

Analysis of putative target genes and $G O$ analysis of troxerutin-specific miRNAs using bioinformatics. From the detection of global miRNA expression levels, our results identified 68 troxerutin-specific miRNAs. miRNAs regulate biological functions through the interference of their target genes (29). To identify biological functions of the miRNAs, we used TargetScan to predict putative target genes of troxerutinspecific miRNAs in UVB-exposed HaCaT cells. Using a seed sequence-based target prediction system (TargetScan), we analyzed the 5 miRNAs upregulated by troxerutin and identified 1,755 putative target genes. For the 63 downregulated miRNAs, 13,768 putative target genes were identified. We then analyzed the categorized functions, using GO analysis within the putative target pool. Following classification into GO categories, we selected significant GO categories using 
Table I. Upregulated and downregulated miRNAs in UVB-exposed HaCaT cells pre-treated with troxerutin.

\begin{tabular}{|c|c|c|c|c|c|}
\hline miRNA & Fold change & miRNA & Fold change & miRNA & Fold change \\
\hline \multicolumn{6}{|c|}{ Upregulated miRNA } \\
\hline hsa-miR-205-3p & 4.29 & hsa-miR-34b-5p & 2.65 & hsa-miR-181a-2-3p & 2.00 \\
\hline hsa-miR-21-3p & 2.97 & hsa-miR-125a-5p & 2.62 & & \\
\hline \multicolumn{6}{|c|}{ Downregulated miRNA } \\
\hline hsa-miR-513b & -23.10 & hsa-miR-630 & -4.88 & hsa-miR-3141 & -3.15 \\
\hline hsa-miR-483-5p & -16.61 & hsa-miR-188-5p & -4.83 & hsa-miR-1915-3p & -3.08 \\
\hline hsa-miR-3648 & -11.55 & hsa-miR-1202 & -4.78 & hsa-miR-1181 & -3.02 \\
\hline hsa-miR-513a-5p & -9.66 & hsa-miR-1224-5p & -4.66 & hsa-miR-940 & -2.98 \\
\hline hsa-miR-135a-3p & -9.49 & hsa-miR-4299 & -4.58 & hsa-miR-320c & -2.97 \\
\hline hsa-miR-572 & -9.26 & hsa-miR-1207-5p & -4.45 & hsa-miR-324-3p & -2.96 \\
\hline hsa-miR-575 & -8.01 & hsa-miR-3663-3p & -4.44 & hsa-miR-3156-5p & -2.89 \\
\hline hsa-miR-4327 & -7.32 & hsa-miR-3162-5p & -4.35 & hsa-miR-1288 & -2.87 \\
\hline hsa-miR-874 & -7.15 & hsa-miR-212-3p & -4.14 & hsa-miR-1305 & -2.86 \\
\hline hsa-miR-638 & -6.68 & hsa-miR-3198 & -4.08 & hsa-miR-1973 & -2.78 \\
\hline hsa-miR-4270 & -6.44 & hsa-miR-642b-3p & -3.96 & hsa-miR-155-5p & -2.76 \\
\hline hsa-miR-1246 & -6.27 & hsa-miR-150-3p & -3.90 & hsa-miR-28-5p & -2.59 \\
\hline hsa-miR-3679-5p & -5.78 & hsa-miR-1914-3p & -3.77 & hsa-miR-452-5p & -2.54 \\
\hline hsa-miR-1225-5p & -5.64 & hsa-miR-762 & -3.66 & hsa-miR-378a-5p & -2.48 \\
\hline hsa-miR-3656 & -5.60 & hsa-miR-3196 & -3.62 & hsa-miR-892b & -2.47 \\
\hline hsa-miR-1275 & -5.59 & hsa-miR-3125 & -3.43 & hsa-miR-4298 & -2.40 \\
\hline hsa-miR-494 & -5.53 & hsa-miR-1268a & -3.30 & hsa-miR-3127-5p & -2.38 \\
\hline hsa-miR-2861 & -5.44 & hsa-miR-9-3p & -3.28 & hsa-miR-939-5p & -2.33 \\
\hline hsa-miR-134 & -5.18 & hsa-miR-629-3p & -3.21 & hsa-miR-29a-3p & -2.26 \\
\hline hsa-miR-4271 & -5.03 & hsa-miR-4281 & -3.21 & hsa-miR-584-5p & -2.16 \\
\hline hsa-miR-125a-3p & -4.91 & hsa-miR-3665 & -3.15 & hsa-miR-3659 & -2.08 \\
\hline
\end{tabular}

miRNAs, microRNAs; UVB, ultraviolet B.

Table II. GO analysis of potential target genes of troxerutin-regulated miRNAs.

\begin{tabular}{|c|c|c|c|c|}
\hline Accession no. & GO term & Count & Percentage $(\%)$ & p-value \\
\hline GO:0045449 & Regulation of transcription & 2,050 & 15.01 & $4.83 \mathrm{E}-18$ \\
\hline GO:0007242 & Intracellular signaling cascade & 1,021 & 7.48 & $6.80 \mathrm{E}-15$ \\
\hline GO:0006793 & Phosphate metabolic processes & 792 & 5.80 & $6.75 \mathrm{E}-12$ \\
\hline GO:0008104 & Protein localization & 712 & 5.21 & $1.74 \mathrm{E}-09$ \\
\hline GO:0043067 & Regulation of programmed cell death & 653 & 4.78 & $2.87 \mathrm{E}-08$ \\
\hline GO:0042127 & Regulation of cell proliferation & 642 & 4.70 & $4.15 \mathrm{E}-10$ \\
\hline GO:0007049 & Cell cycle regulation & 611 & 4.47 & 1.69E-05 \\
\hline GO:0006811 & Ion transport & 603 & 4.42 & $3.54 \mathrm{E}-05$ \\
\hline
\end{tabular}

GO, gene ontology; miRNAs, microRNAs.

parameters, such as a count of $>600$, a percentage (\%) of $>4 \%$ and a $\mathrm{p}$-value $<0.01$. Our analyses revealed the following distribution of biological processes: regulation of transcription $(2,050$; number of GO term-related genes in the putative target pool), intracellular signaling cascades $(1,021)$, phosphate metabolic processes (792), protein localization (712), regulation of programmed cell death (653), regulation of cell proliferation (642), cell cycle (611) and ion transport (603) (Table II).
The aforementioned effects of troxerutin were further grouped into four cell functional groups: cell death and apoptosis, cell proliferation and the cell cycle, migration and DNA repair. These groups included the following: cell death and apoptosis, 16 GO terms; cell proliferation and cell cycle, 19 GO terms; migration, 8 GO terms; and DNA repair, 2 GO terms (Table III). Overall, these results demonstrated that troxerutin exerted protective effects against UVB-induced damage, 
Table III. Groupring of GO terms into four cell functional groups reflecting the effects of troxerutin.

\begin{tabular}{|c|c|c|c|c|}
\hline Accession no. & GO term & Count & Percentage $(\%)$ & $\mathrm{p}$-value \\
\hline \multicolumn{5}{|c|}{ Cell death and apoptosis (16 GO terms) } \\
\hline GO:0043067 & Regulation of programmed cell death & 653 & 4.78 & 2.87E-08 \\
\hline GO:0010941 & Regulation of cell death & 653 & 4.78 & 8.51E-08 \\
\hline GO:0042981 & Regulation of apoptosis & 647 & 4.74 & $2.75 \mathrm{E}-08$ \\
\hline GO:0016265 & Death & 569 & 4.17 & 4.95E-05 \\
\hline GO:0008219 & Cell death & 564 & 4.13 & 7.79E-05 \\
\hline GO:0012501 & Programmed cell death & 474 & 3.47 & $1.65 \mathrm{E}-03$ \\
\hline GO:0006915 & Apoptosis & 466 & 3.41 & $2.44 \mathrm{E}-03$ \\
\hline GO:0043068 & Positive regulation of programmed cell death & 344 & 2.52 & 4.31E-04 \\
\hline GO:0010942 & Positive regulation of cell death & 344 & 2.52 & 7.94E-04 \\
\hline GO:0043065 & Positive regulation of apoptosis & 341 & 2.50 & $5.82 \mathrm{E}-04$ \\
\hline GO:0043069 & Negative regulation of programmed cell death & 289 & 2.12 & $2.55 \mathrm{E}-04$ \\
\hline GO:0060548 & Negative regulation of cell death & 289 & 2.12 & $3.66 \mathrm{E}-04$ \\
\hline GO:0012502 & Induction of programmed cell death & 251 & 1.84 & $1.16 \mathrm{E}-02$ \\
\hline GO:0006917 & Induction of apoptosis & 250 & 1.83 & $1.27 \mathrm{E}-02$ \\
\hline GO:0006916 & Anti-apoptosis & 162 & 1.19 & $3.08 \mathrm{E}-02$ \\
\hline GO:0043524 & Negative regulation of neuron apoptosis & 49 & 0.36 & $8.44 \mathrm{E}-05$ \\
\hline \multicolumn{5}{|c|}{ Cell proliferation and cell cycle (19 GO terms) } \\
\hline GO:0042127 & Regulation of cell proliferation & 642 & 4.70 & $4.15 \mathrm{E}-10$ \\
\hline GO:0007049 & Cell cycle & 611 & 4.47 & $1.69 \mathrm{E}-05$ \\
\hline GO:0022402 & Cell cycle process & 442 & 3.24 & $7.41 \mathrm{E}-04$ \\
\hline GO:0008283 & Cell proliferation & 345 & 2.53 & $7.21 \mathrm{E}-04$ \\
\hline GO:0008284 & Positive regulation of cell proliferation & 336 & 2.46 & $2.11 \mathrm{E}-05$ \\
\hline GO:0022403 & Cell cycle phase & 331 & 2.42 & $2.36 \mathrm{E}-04$ \\
\hline GO:0008285 & Negative regulation of cell proliferation & 292 & 2.14 & $1.24 \mathrm{E}-04$ \\
\hline GO:0040008 & Regulation of growth & 265 & 1.94 & $1.61 \mathrm{E}-02$ \\
\hline GO:0000279 & M phase & 260 & 1.90 & $3.88 \mathrm{E}-03$ \\
\hline GO:0051726 & Regulation of cell cycle & 260 & 1.90 & $6.85 \mathrm{E}-03$ \\
\hline GO:0000087 & M phase of mitotic cell cycle & 173 & 1.27 & $6.99 \mathrm{E}-02$ \\
\hline GO:0001558 & Regulation of cell growth & 151 & 1.11 & $6.26 \mathrm{E}-02$ \\
\hline GO:0010564 & Regulation of cell cycle process & 96 & 0.70 & $3.86 \mathrm{E}-03$ \\
\hline GO:0007050 & Cell cycle arrest & 87 & 0.64 & $5.25 \mathrm{E}-03$ \\
\hline GO:0051329 & Interphase of mitotic cell cycle & 85 & 0.62 & $1.91 \mathrm{E}-02$ \\
\hline GO:0051327 & M phase of meiotic cell cycle & 78 & 0.57 & $9.53 \mathrm{E}-02$ \\
\hline GO:0045927 & Positive regulation of growth & 66 & 0.48 & 4.23E-02 \\
\hline GO:0000082 & G1/S transition of mitotic cell cycle & 49 & 0.36 & $1.34 \mathrm{E}-02$ \\
\hline GO:0045787 & Positive regulation of cell cycle & 47 & 0.34 & $9.65 \mathrm{E}-02$ \\
\hline \multicolumn{5}{|c|}{ Migration (8 GO terms) } \\
\hline GO:0048870 & Cell motility & 244 & 1.79 & $3.03 \mathrm{E}-03$ \\
\hline GO:0016477 & Cell migration & 227 & 1.66 & $1.11 \mathrm{E}-04$ \\
\hline GO:0042060 & Wound healing & 151 & 1.11 & $2.78 \mathrm{E}-02$ \\
\hline GO:0030334 & Regulation of cell migration & 143 & 1.05 & $2.08 \mathrm{E}-04$ \\
\hline GO:0030335 & Positive regulation of cell migration & 79 & 0.58 & $3.86 \mathrm{E}-04$ \\
\hline GO:0030336 & Negative regulation of cell migration & 48 & 0.35 & $5.24 \mathrm{E}-02$ \\
\hline GO:0010595 & Positive regulation of endothelial cell migration & 13 & 0.10 & 8.71E-02 \\
\hline GO:0002685 & Regulation of leukocyte migration & 19 & 0.14 & $5.47 \mathrm{E}-02$ \\
\hline \multicolumn{5}{|c|}{ DNA repair (2 GO terms) } \\
\hline GO:0051052 & Regulation of DNA metabolic process & 90 & 0.66 & $9.55 \mathrm{E}-02$ \\
\hline GO:0051054 & Positive regulation of DNA metabolic process & 47 & 0.35 & $5.15 \mathrm{E}-02$ \\
\hline
\end{tabular}

GO, gene ontology. 
which was related to an alteration in cellular miRNA expression profiles.

\section{Discussion}

In the skin, UVB radiation is a crucial inducer of apoptosis and growth arrest (5). In the epidermis, the accumulation of UVB-induced damaged keratinocytes results in sunburn, psoriasis and skin cancer (30). Therefore, protection from UVB-induced keratinocyte damage, including cell death, growth arrest, DNA damage and decreased migration, is essential in order to maintain epidermal homeostasis.

In the present study, we demonstrated that troxerutin protected cells from UVB-induced decrease in cell growth through the suppression of apoptosis. Pre-treatment with troxerutin protected the $\mathrm{HaCaT}$ cells from UVB-induced growth arrest (Fig. 2) by preventing apoptosis (Fig. 3). Also, migration assay results deonstrated that troxerutin increased cell migration activity (Fig. 4). In previous studies, troxerutininduced DNA repair enhanced its protective effects against gamma radiation $(28,30)$. Similarly, in the present study, DNA repair activity was increased by troxerutin in $\mathrm{HaCaT}$ cells (Fig. 5). In addition, using miRNA microarray analysis and bioinformatics tools, we identified specific troxerutininduced miRNAs and found a link between troxerutin-induced miRNAs, as well as anti-apoptotic, enhanced migration and DNA repair effects. In UVB-exposed $\mathrm{HaCaT}$ cells, troxerutin altered the expression of 68 miRNAs, with a $\geq 2$-fold up- or downregulation (Fig. 3).

In our study, in UVB-exposed HaCaT cells, miR-181a-5p was upregulated 2-fold by troxerutin. The miR-181a, a mature form of miR-181a-1, and miR-181a-2, have been implicated in proliferation, migration and invasion, and target $B I M$, a member of the apoptotic BCL-2 family (32,33). In the present study, in the downregulated miRNA group, miR-513a-5p was markedly decreased by 9.66 -fold in troxerutin-treated HaCaT cells. This miRNA induces apoptosis by targeting the X-linked inhibitor of apoptosis (XIAP) in endothelial cells (34). miR-874 was also decreased by 7.15 -fold in troxerutin-treated $\mathrm{HaCaT}$ cells and has been shown to contribute to cell proliferation through its effects on histone deacetylase 1 (35). miR-874 has been implicated in cell proliferation and migration by targeting aquaporin-3 (36). In this study, the expression levels of miR-1246 and miR-494 were significantly decreased in troxerutin-pre-treated $\mathrm{HaCaT}$ cells exposed to UVB. The expression of miR-1246 has been reported to be regulated by $\mathrm{p} 53$, a crucial transcription factor in the DNA damage response, and can repress the translation of dual-specificity tyrosine-(Y)-phosphorylation regulated kinase 1A (DYRK1A), an inducer of proliferation (37). miR-494 has been implicated in TRAIL-induced apoptosis through the repression of BIM (38). In addition, miR-494 regulates three pro-apoptotic proteins [PTEN, Rho-associated, coiled-coil containing protein kinase 1 (ROCK1) and $\mathrm{Ca}^{2+} /$ calmodulindependent protein kinase $\delta$ CaMKII $\delta$ ] and two anti-apoptotic proteins [fibroblast growth factor receptor 2 (FGFR2) and leukemia inhibitory factor (LIF)] (39). Therefore, our results suggest that troxerutin protects cells from UVB-induced DNA damage and apoptosis by regulating miRNA expression.

Furthermore, we predicted target genes of troxerutinregulated miRNAs and analyzed the GO terms of potential target genes using DAVID bioinformatics resources. DAVID functional annotation suggested that the target genes of troxerutin-regulated miRNAs have a regulatory role in transcription, intracellular signaling cascades, phosphate metabolic processes, protein localization, programmed cell death, cell proliferation, the cell cycle and ion transport (Table II). Indeed, we grouped the GO terms into four functional groups induced by troxerutin in UVB-damaged cells: cell death and apoptosis, cell proliferation and the cell cycle, cell migration and DNA repair (Table III).

Overall, the current study provides evidence of the protective effects of troxerutin against UVB-induced damage in $\mathrm{HaCaT}$ cells. The present study demonstrates a significant correlation between the four cell function groups with alterations in miRNA expression, troxerutin-regulated miRNA function, and GO analysis of the miRNA target genes.

\section{Acknowledgements}

We would like to thank all other members of Coreana Cosmetics Co., Ltd. for their support. This study was supported by the KU Research Professor Program of Konkuk University and a grant from the Ministry of Science, ICT and Future Planning (no. 20110028646) of the Republic of Korea.

\section{References}

1. Oresajo C, Pillai S, Manco M, Yatskayer M and McDaniel D: Antioxidants and the skin: understanding formulation and efficacy. Dermatol Ther 25: 252-259, 2012.

2. Campbell C, Quinn AG, Angus B, Farr PM and Rees JL: Wavelength specific patterns of p53 induction in human skin following exposure to UV radiation. Cancer Res 53: 2697-2699, 1993.

3. de Gruijl FR and Van der Leun JC: Estimate of the wavelength dependency of ultraviolet carcinogenesis in humans and its relevance to the risk assessment of a stratospheric ozone depletion. Health Phys 67: 319-325, 1994.

4. Chainiaux F, Magalhaes JP, Eliaers F, Remacle J and Toussaint O: UVB-induced premature senescence of human diploid skin fibroblasts. Int J Biochem Cell Biol 34: 1331-1339, 2002.

5. Matsumura Y and Ananthaswamy HN: Toxic effects of ultraviolet radiation on the skin. Toxicol Appl Pharmacol 195: 298-308, 2004.

6. Tanaka M, Koyama Y and Nomura Y: Effects of collagen peptide ingestion on UV-B-induced skin damage. Biosci Biotechnol Biochem 73: 930-932, 2009.

7. Everett MA, Yeargers E, Sayre RM, Olson RL: Penetration of epidermis by ultraviolet rays. Photochem Photobiol 5: 533-542, 1966.

8. Suter MM, Schulze K, Bergman W, Welle M, Roosje P and Müller EJ: The keratinocyte in epidermal renewal and defense. Vet Dermatol 20: 515-532, 2009.

9. Kulms D, Zeise E, Pöppelmann B and Schwarz T: DNA damage, death receptor activation and reactive oxygen species contribute to ultraviolet radiation-induced apoptosis in an essential and independent way. Oncogene 21: 5844-5851, 2002.

10. Bode AM and Dong Z: Mitogen-activated protein kinase activation in UV-induced signal transduction. Sci STKE 2003: RE2, 2003.

11. Assefa Z, Van Laethem A, Garmyn M and Agostinis P: Ultraviolet radiation-induced apoptosis in keratinocytes: on the role of cytosolic factors. Biochim Biophys Acta 1755: 90-106, 2005.

12. Rezvani HR, Mazurier F, Cario-André M, et al: Protective effects of catalase overexpression on UVB-induced apoptosis in normal human keratinocytes. J Biol Chem 281: 17999-18007, 2006.

13. Henseleit U, Zhang J, Wanner R, Haase I, Kolde G and Rosenbach T: Role of p53 in UVB-induced apoptosis in human HaCaT keratinocytes. J Invest Dermatol 109: 722-727, 1997. 
14. Enk CD, Jacob-Hirsch J, Gal H, et al: The UVB-induced gene expression profile of human epidermis in vivo is different from that of cultured keratinocytes. Oncogene 25: 2601-2614, 2006.

15. Zhou BR, Xu Y, Permatasari F, et al: Characterization of the miRNA profile in UVB-irradiated normal human keratinocytes. Exp Dermatol 21: 317-319, 2012.

16. Zhou BR, Xu Y and Luo D: Effect of UVB irradiation on microRNA expression in mouse epidermis. Oncol Lett 3 : 560-564, 2012

17. Guo Z, Zhou B, Liu W, et al: MiR-23a regulates DNA damage repair and apoptosis in UVB-irradiated HaCaT cells. J Dermatol Sci 69: 68-76, 2013.

18. Li W, Di W, Hua L, Zhou B, Guo Z and Luo D: UVB suppresses PTEN expression by upregulating miR-141 in HaCaT cells. J Biomed Res 25: 135-140, 2011.

19. Pothof J, Verkaik NS, van IJcken W, et al: MicroRNA-mediated gene silencing modulates the UV-induced DNA-damage response. EMBO J 28: 2090-2099, 2009.

20. Fan SH,Zhang ZF, Zheng YL, et al: Troxerutin protects the mouse kidney from $D$-galactose-caused injury through anti-inflammation and anti-oxidation. Int Immunopharmacol 9: 91-96, 2009.

21. Zhang ZF, Fan SH, Zheng YL, et al: Troxerutin protects the mouse liver against oxidative stress-mediated injury induced by D-galactose. J Agric Food Chem 57: 7731-7736, 2009.

22. $\mathrm{Lu} \mathrm{J}, \mathrm{Wu} \mathrm{DM}, \mathrm{Hu} \mathrm{B}$, et al: Chronic administration of troxerutin protects mouse brain against D-galactose-induced impairment of cholinergic system. Neurobiol Learn Mem 93: 157-164, 2010.

23. $\mathrm{Lu} \mathrm{J}, \mathrm{Wu} \mathrm{DM}, \mathrm{Hu} \mathrm{B}$, Zheng YL, Zhang ZF and Wang YJ: NGF-dependent activation of TrkA pathway: a mechanism for the neuroprotective effect of troxerutin in D-galactose-treated mice. Brain Pathol 20: 952-965, 2010.

24. Lu J, Wu DM, Zhang ZF, Zheng YL, Hu B, and Zhang ZF: troxerutin protects against high cholesterol-induced cognitive deficits in mice. Brain 134: 783-797, 2011

25. Budzianowski J, Korzeniowska K, Chmara E and Mrozikiewicz A: Microvascular protective activity of flavonoid glucuronides fraction from Tulipa gesneriana. Phytother Res 13: 166-168, 1999

26. Boisseau MR, Taccoen A, Garreau C, Vergnes C, Roudaut MF and Garreau-Gomez B: Fibrinolysis and hemorheology in chronic venous insufficiency: A double blind study of troxerutin efficiency. J Cardiovasc Surg (Torino) 36: 369-374, 1995.

27. Ping X, Junqing J, Junfeng J and Enjin J: Radioprotective effects of troxerutin against gamma irradiation in V79 cells and mice. Asian Pac J Cancer Prev 12: 2593-2596, 2011.
28. Maurya DK, Balakrishnan S, Salvi VP and Nair CK: Protection of cellular DNA from gamma-radiation-induced damages and enhancement in DNA repair by troxerutin. Mol Cell Biochem 280: 57-68, 2005.

29. Ambros V. microRNAs: tiny regulators with great potential. Cell 107: 823-826, 2001.

30. Raj D, Brash DE and Grossman D: Keratinocyte apoptosis in epidermal development and disease. J Invest Dermatol 126: 243-257, 2006

31. Maurya DK, Salvi VP and Krishnan Nair CK: Radioprotection of normal tissues in tumor-bearing mice by troxerutin. J Radiat Res 45: 221-228, 2004.

32. Taylor MA, Sossey-Alaoui K, Thompson CL, Danielpour D and Schiemann WP: TGF- $\beta$ upregulates miR-181a expression to promote breast cancer metastasis. J Clin Invest 123: 150-163, 2013.

33. Li S, Niu X, Cui A, He Y and Wu W: The effects of miR-181a on proliferation, migration and invasion abilities of esophageal carcinoma cell line TE11. Tumor 31: 613-618, 2011.

34. Shin S, Moon KC, Park KU and Ha E: MicroRNA-513a-5p mediates TNF- $\alpha$ and LPS induced apoptosis via downregulation of X-linked inhibitor of apoptotic protein in endothelial cells. Biochimie 94: 1431-1436, 2012.

35. Nohata N, Hanazawa T, Kinoshita T, et al: Tumour-suppressive microRNA-874 contributes to cell proliferation through targeting of histone deacetylase 1 in head and neck squamous cell carcinoma. Br J Cancer 108: 1648-1658, 2013.

36. Jiang B, Li Z, Zhang W, et al: miR-874 Inhibits cell proliferation, migration and invasion through targeting aquaporin-3 in gastric cancer. J Gastroenterol: June 26, 2013 (Epub ahead of print).

37. Zhang Y, Liao JM, Zeng SX and Lu H: p53 downregulates Down syndrome-associated DYRK1A through miR-1246. EMBO Rep 12: 811-817, 2011

38. Romano G, Acunzo M, Garofalo M, et al: MiR-494 is regulated by ERK $1 / 2$ and modulates TRAIL-induced apoptosis in nonsmall-cell lung cancer through BIM down-regulation. Proc Natl Acad Sci USA 109: 16570-16575, 2012.

39. Wang X, Zhang X, Ren XP, et al: MicroRNA-494 targeting both proapoptotic and antiapoptotic proteins protects against ischemia/reperfusion-induced cardiac injury. Circulation 122 1308-1318, 2010. 